\title{
Myocardial perfusion imaging with real-time respiratory triggering: Impact of inspiration breath-hold on left ventricular functional parameters
}

\author{
Ronny R. Buechel, MD, ${ }^{a}$ Lars Husmann, MD, ${ }^{a}$ Aju P. Pazhenkottil, MD, ${ }^{a}$ \\ Rene Nkoulou, MD, ${ }^{a}$ Bernhard A. Herzog, MD, ${ }^{a}$ Irene A. Burger, ${ }^{a}$ \\ Jelena R. Ghadri, MD, ${ }^{a}$ Mathias Wolfrum, MD, ${ }^{a}$ and Philipp A. Kaufmann, $M D^{a, b}$
}

Background. The latest gamma-camera generation with cadmium-zinc-telluride (CZT) detectors allows myocardial perfusion imaging (MPI) with respiratory triggering at breathhold. We assessed its impact on functional left ventricular (LV) parameters.

Methods. Twenty-eight consecutive patients underwent a one-day ${ }^{99} \mathrm{~m}$ Tc-tetrofosmin pharmacologic stress/rest imaging protocol on a novel CZT camera. Electrocardiogram-gated high-dose (rest) MPI was performed without and with real-time respiratory triggering by intermittent scanning confined to breath-hold at deep inspiration. We studied the effect of respiratory triggering at deep inspiration levels on $L V$ wall motion, wall thickening, $L V$ volumes and ejection fraction (LVEF) compared to regular MPI without respiratory triggering.

Results. Compared to regular MPI without respiratory triggering, systolic and diastolic LV volumes and stroke volumes decreased significantly $(P<0.05)$ when respiratory triggering was applied. By contrast, there was no significant change in LVEF, with a high correlation $(r=.939, P<0.001)$ between the two measurements. Furthermore, respiratory triggering introduced a significant change $(P<0.05)$ in regional $L V$ wall motion.

Conclusions. Respiratory-triggered MPI with breath-hold at deep inspiration levels introduces significant changes to the measured $L V$ volumes, stroke volumes and regional wall motion but does not significantly affect global LVEF when compared to regular MPI with normal breathing. (J Nucl Cardiol 2010;17:848-52.)

Key Words: Myocardial perfusion imaging $\cdot$ SPECT $\cdot$ left ventricular function $\cdot$ gated SPECT

\section{INTRODUCTION}

A few years ago, retrospective respiratory gating has been first introduced to single photon emission computed tomography myocardial perfusion imaging

From the Cardiac Imaging, ${ }^{\text {a }}$ University Hospital Zurich, Zurich, Switzerland; Zurich Center for Integrative Human Physiology (ZIHP), ${ }^{\mathrm{b}}$ University of Zurich, Zurich, Switzerland

The University Hospital Zurich holds a research grant with GE Healthcare.

Received for publication Dec 30, 2009; final revision accepted Apr 1, 2010.

Reprint requests: Philipp A. Kaufmann, MD, Cardiac Imaging, University Hospital Zurich, Ramistrasse 100, 8091 Zurich, Switzerland; pak@usz.ch.

$1071-3581 / \$ 34.00$

Copyright (C) 2010 by the American Society of Nuclear Cardiology. doi:10.1007/s12350-010-9232-4
(SPECT-MPI). ${ }^{1,2}$ Recent studies have demonstrated its potential ability to improve the quality of SPECT-MPI by reducing respiratory-related motion artefacts ${ }^{3-6}$ andif performed at deep inspiration levels-spill-over effects due to sub-diaphragmatic tracer activity. ${ }^{7}$ Thus, respiratory gating has been suggested to improve MPI quality but has remained challenging due to the step-and-shoot acquisition mode in SPECT gamma cameras with rotating detectors. This may have contributed to prevent the more widespread use of this promising technique.

The introduction of a new generation of gamma camera detectors using cadmium-zinc-telluride (CZT) semiconductors has enabled a substantial miniaturization of the detectors, which allows their alignment around the patient, rendering camera rotation unnecessary. This not only reduces the required scan time, ${ }^{8,9}$ but also foremost allows breath-hold acquisition with the potential benefits mentioned above. However, the 
impact of deep inspiration breath-hold on left ventricular (LV) function obtained from gated SPECT is unknown.

The effects of respiration on $\mathrm{LV}$ function are numerous and have been the subject of several extensive studies. $^{10-19}$ Previous studies evaluating respiratory gating in nuclear MPI, however, have mainly focused on its effect on myocardial tracer uptake but not on LV function. Thus, the aim of this study was to assess the impact of scanning at deep inspiration breath-hold on functional LV parameters extracted from MPI.

\section{METHODS}

\section{Study Protocol and Image Acquisition}

Twenty-eight consecutive patients referred to SPECTMPI for exclusion of coronary artery disease (CAD) were included in this study.

All patients underwent a one-day ${ }^{99 \mathrm{~m}} \mathrm{Tc}$-tetrofosmin pharmacological stress/rest MPI protocol as suggested by the guidelines of the European Association of Nuclear Medicine. ${ }^{20}$ Pharmacological stress was induced by standard adenosine (in 27 patients; 96\%) or dobutamine (in 1 patient; 4\%) infusion. ${ }^{99 \mathrm{~m}} \mathrm{Tc}$-tetrofosmin injection (mean dose $328 \pm 24 \mathrm{MBq}$; range 292-414 MBq) was administered after 3 minutes of induced stress. Injection was followed by a waiting time according to the guidelines ${ }^{21}$ before image acquisition. Rest MPI was performed thereafter with the identical acquisition protocol after injection of a three times higher dose of ${ }^{99 \mathrm{~m}} \mathrm{Tc}$-tetrofosmin (mean dose $941 \pm 51 \mathrm{MBq}$; range $900-1180 \mathrm{MBq}$ ) on an ultrafast CZT camera (Discovery $530 \mathrm{NMc}$, GE Healthcare) once with regular acquisition, i.e. with normal breathing, and a second time with real-time respiratory triggering. To this aim, patients were repeatedly asked to perform a breath-hold at a deep inspiration-level while scanning was manually initiated after end-inspiration to ensure confinement of acquisition to breath-hold at deep inspiration. After scanning at breath-hold for a duration (10-20 seconds) which had been individually determined according to the patient's ability, acquisition was manually interrupted and the patient was allowed to breathe normally before continuing the scan. This sequence was repeated until an overall scan duration of 3 minutes for stress and 2 minutes for rest was reached as previously established for this camera. ${ }^{8,9}$

The CZT camera uses a multi-pinhole collimator (effective diameter aperture of $5.1 \mathrm{~mm}$ ) and 19 stationary detectors simultaneously imaging 19 views of the heart. Each detector contains $32 \times 32$ pixillated $(2.46 \times 2.46 \mathrm{~mm})$ CZT elements. A $10 \%$ symmetric energy window at $140 \mathrm{keV}$ was used. Image acquisition was performed using list mode and ECG-gated scans were acquired using 16 bins. Scan time was 3 minutes for stress and 2 minutes for rest, as previously established. ${ }^{8}$ As the CZT detectors are aligned around the patient, covering the entire heart, acquisition of all cardiac views simultaneously is possible.

The study protocol was approved by the local ethics committee and written informed consent was obtained from every patient.

\section{MPI Reconstruction and Quantitative Analysis}

All scans were reconstructed on a dedicated workstation using an iterative algorithm with Maximum Likelihood Expectation Maximization. The software packages Myovation for Alcyone (GE Healthcare) and QGS/QPS (Cedars-Sinai Medical Center) were used for analysis. Quantitative analysis was performed on the ECG-gated high-dose (rest) MPI to determine LV wall motion, wall thickening (projected on 20 segment polar maps), LV volumes and ejection fraction (LVEF). Mean HR during acquisition was recorded for each scan.

\section{Statistical Methods}

SPSS 17.0 (SPSS Inc.) was used for statistical testing. Quantitative variables were expressed as mean \pm standard deviation and categorical variables as frequencies or percentages. Wilcoxon rank-sum test and paired-samples $t$-test were used for non-parametric and parametric data, respectively, to test for differences between parameters acquired during the scans. Similarly, Spearman or Pearson analysis was applied to assess correlations. $P$-values of less than 0.05 were considered statistically significant.

\section{RESULTS}

Patient characteristics are given in Table 1. Realtime respiratory triggering was successfully performed in all patients over a wide range of LVEF $(32 \%$ to $82 \%)$.

Mean values for volumes and LVEF with and without respiratory triggering are given in Table 2. Respiratory triggering introduced a significant decrease in LV volumes and stroke volumes (Figures 1 and 2). By contrast, LVEF obtained from respiratory-triggered MPI was not affected and correlated highly with LVEF extracted from regular MPI (Figure 3). Nevertheless, one patient met criteria ${ }^{22}$ for $\mathrm{LV}$ dilatation according to end-systolic (ESV) and end-diastolic volumes (EDV) extracted from MPI without respiratory triggering (ESV: $86 \mathrm{~mL}$, EDV: $144 \mathrm{~mL}$ ) but did not meet these criteria when respiratory triggering was applied (ESV: $77.1 \mathrm{~mL}$; EDV: $110 \mathrm{~mL}$ ) despite comparable EF in both scans.

Overall analysis of LV wall motion revealed significant $(P<0.05)$ differences between MPI with and without respiratory triggering. Per segment analysis documented regional differences with reduced wall motion in the antero-septal wall, compensated by an increase in the inferior wall motion when comparing respiratory triggered with regular MPI (Figure 4). By contrast, there was no significant difference in regional wall thickening between MPI with and without respiratory triggering. 
Table 1. Patient baseline characteristics $(N=28)$

\begin{tabular}{ll}
\hline Male & $17(61 \%)$ \\
Age (years) & \\
Mean \pm SD & $67 \pm 10$ \\
Range & $44-79$ \\
BMI $\left(\mathrm{kg} / \mathrm{m}^{2}\right)$ & \\
Mean \pm SD & $26 \pm 3$ \\
Range & $20-33$ \\
Cardiovascular risk factors & \\
Obesity (BMI $\left.>30 \mathrm{~kg} / \mathrm{m}^{2}\right)$ & $5(18 \%)$ \\
Smoking & $6(21 \%)$ \\
Diabetes mellitus & $3(11 \%)$ \\
Hypertension & $21(75 \%)$ \\
Dyslipidemia & $14(50 \%)$ \\
Positive family history & $5(18 \%)$ \\
Reason for referral & \\
Suspected CAD & $16(57 \%)$ \\
Follow-up of known CAD & $11(39 \%)$ \\
Pre-OP assessment & $1(4 \%)$ \\
Previous cardiac events & \\
Revascularisation & $11(39 \%)$ \\
Myocardial infarction & $6(21 \%)$
\end{tabular}

$S D$, Standard deviation; $B M I$, body mass index; $C A D$, coronary artery disease; pre-OP assessment, preoperative assessment before non-cardiac surgery.

\section{DISCUSSION}

The recent introduction of CZT gamma cameras with its impressive detector miniaturization allows an alignment of the detectors around the patient which renders the need for camera rotation unnecessary and therefore substantially reduces scan times. ${ }^{8,9}$ Thus, these improvements compared to conventional dualhead SPECT cameras may pave the way for a more wide-spread use of respiratory triggering. The latter has been shown to improve MPI quality by reducing respiratory-related motion artefacts. ${ }^{3,4}$ Furthermore, if performed at deep inspiration levels, respiratory triggering may-similar to the well-established effect of prone position on MPI—help to reduce artefacts caused

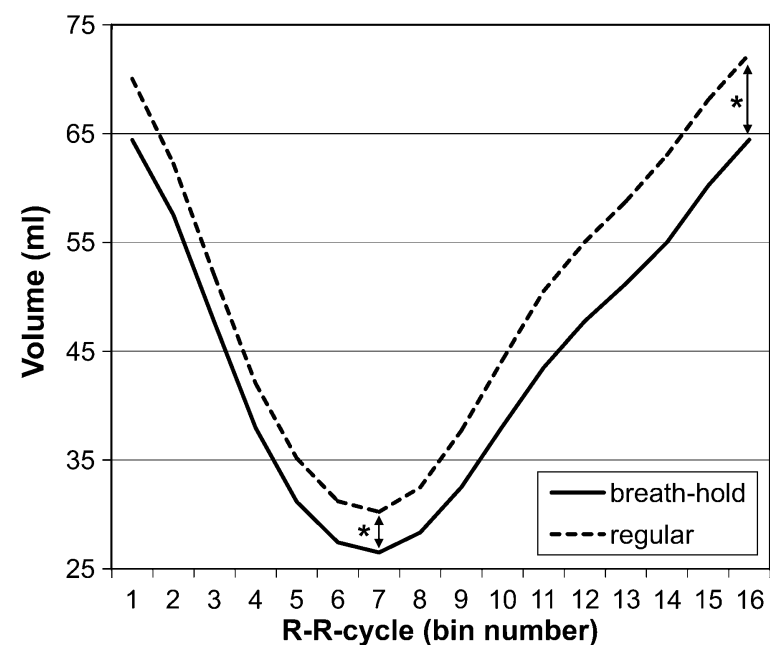

Figure 1. Comparison of LV volumes acquired during ECGgated high-dose scans with (breath-hold, solid line) and without (regular, dotted line) respiratory triggering showed a significant $(* P<0.005)$ difference.

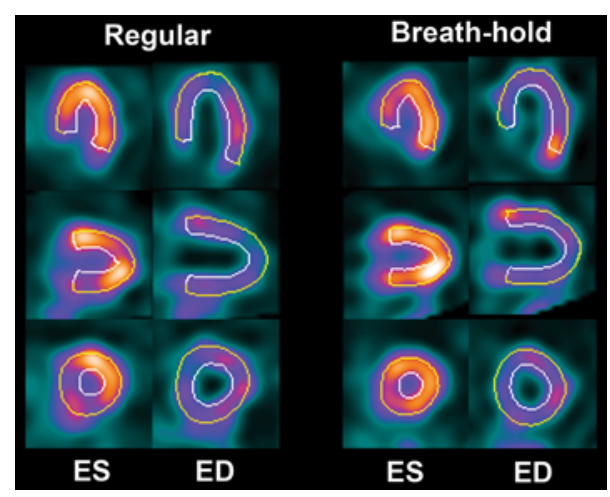

Figure 2. End-diastolic $(E S)$ and end-systolic $(E S)$ left ventricle during regular breathing and at deep inspiration breath-hold do reveal a small but visually recognizable difference in ventricle size. Borders of automatic edge detection are depicted.

by soft-tissue attenuation or spill-over effects due to subdiaphragmatic tracer activity.

This study assesses the impact of such respiratory triggering at deep inspiration levels on LV function parameters extracted from MPI. In line with previous

Table 2. Mean values of functional parameters derived from MPI with and without respiratory triggering $(\mathrm{N}=28)$

\begin{tabular}{lcccc}
\hline & EDV $(\mathbf{m L})$ & ESV $(\mathbf{m L})$ & SV $(\mathbf{m L})$ & LVEF (\%) \\
\hline Regular MPI & $72 \pm 22$ & $30 \pm 20$ & $42 \pm 9$ & $62 \pm 13$ \\
Respiratory triggered MPI & $65 \pm 22$ & $27 \pm 18$ & $39 \pm 7$ & $62 \pm 12$ \\
$P$-value & $<0.005$ & $<0.005$ & $<0.05$ & NS \\
\hline
\end{tabular}

NS, Non significant. 

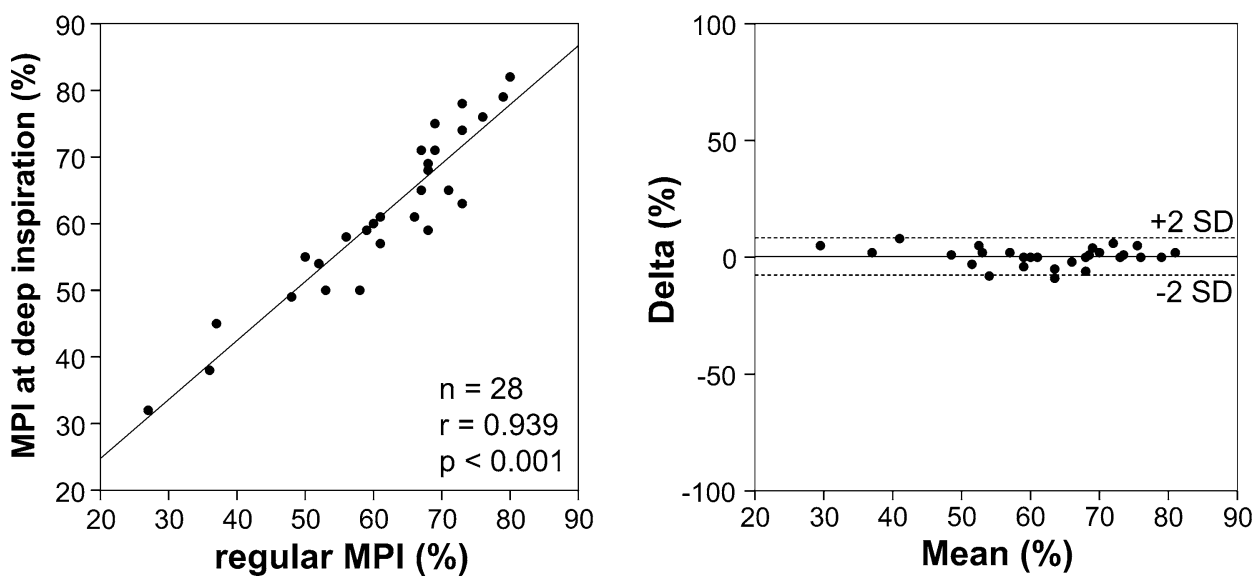

Figure 3. LV ejection fraction extracted from MPI with and without respiratory triggering correlated highly $(r=.939)$. Similarly, Bland-Altman limits of agreement were narrow $(-7 \%$ to $8 \%)$.

A

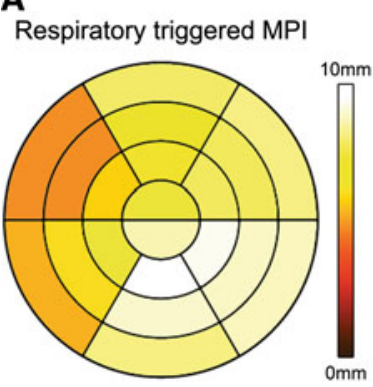

B

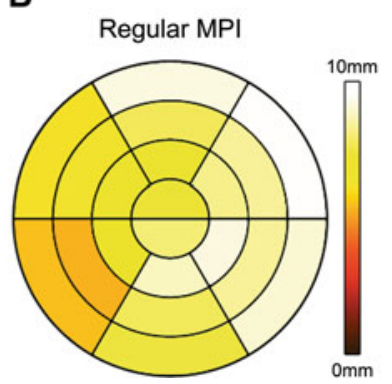

C Respiratory triggered vs. regular MPI

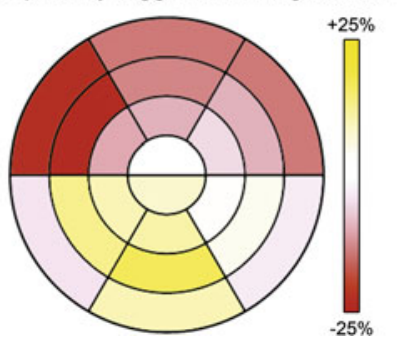

Figure 4. Per segment analysis of wall motion extracted from electrocardiogram-gated MPI with (A) and without respiratory triggering $(\mathbf{B})$. The percentage difference plot (C) reveals that inspiration breath-hold is associated with a decrease in antero-septal wall motion, compensated by an increase in the inferior segments.

reports using echocardiography, ${ }^{11,15,16}$ cardiac catheterization, ${ }^{10,12,13}$ and nuclear imaging, ${ }^{14,19} \mathrm{LV}$ volumes decreased at inspiration. Interestingly, however, the present data reveal no change in LVEF, contrary to some of the previous reports. ${ }^{14,19}$ This may be due to the fact that previous studies have compared LVEF between expiration and inspiration, whereas in this study a comparison between regular breathing and breath-hold at deep inspiration levels was performed, leading to less pronounced differences.

Our results confirm that LVEF values obtained from scanning with respiratory triggering at deep inspiration levels can be reliably used as the values are interchangeable with those obtained from regular MPI. By contrast, absolute LV volumes are not interchangeable and therefore must be judged with caution when obtained at deep inspiration, as the latter decreases the volumes.
It is a well-known fact that respiration may affect diastolic filling and systolic ejection. Factors such as changes in LV transmural pressures and decreased LV preload have been identified as mechanisms contributing to variations in the ventricular volumes with inspiration. Furthermore, several studies suggest that an increase in right ventricular volume may cause a decrease in LV compliance through ventricular interdependence. ${ }^{11,13,14}$ This may to some extent explain the relative decrease in antero-septal LV wall motion found in this study during breath-hold at deep inspiration.

While previous reports have focused mainly on how respiratory triggering affects myocardial tracer uptake, our study is the first to demonstrate its effects on LV functional parameters of patients. Importantly, when compared to regular MPI, respiratory triggering yields interchangeable LVEV values but introduced significant changes regarding LV volumes and wall motion. The 
magnitude of these changes should be taken into account when evaluating respiratory-triggered MPI studies.

It may be perceived as a limitation that in this study a comparison was conducted between breath-hold at deep inspiration levels and regular breathing (i.e. an average of regular inspiration and expiration) but not between deep inspiration and forced expiration. However, the aim of this study was to evaluate the effects of respiratory triggering on every-day clinical MPI which is not performed at forced expiration. Therefore, we felt it more adequate to use regular breathing as standard of reference.

\section{CONCLUSION}

Respiratory-triggered MPI with breath-hold at deep inspiration levels introduces significant changes to the measured LV volumes, stroke volumes and wall motion but does not significantly affect LVEF when compared to regular MPI with normal breathing.

\section{Acknowledgments}

This study was supported by a grant from the Swiss National Science Foundation and by the ZIHP (Zurich Center for Integrative Human Physiology, University of Zurich, Switzerland).

\section{References}

1. Cho K, Kumiata S, Okada S, Kumazaki T. Development of respiratory gated myocardial SPECT system. J Nucl Cardiol 1999;6:20-8

2. Segars WP, Tsui BMW. Study of the efficacy of respiratory gating in myocardial SPECT using the new 4-D NCAT. IEEE Trans Nucl Sci 2000;47:1192-5.

3. Kovalski G, Israel O, Keidar Z, Frenkel A, Sachs J, Azhari H. Correction of heart motion due to respiration in clinical myocardial perfusion SPECT scans using respiratory gating. J Nucl Med 2007;48:630-6.

4. Kovalski G, Keidar Z, Frenkel A, Sachs J, Attia S, Azhari H. Dual "motion-frozen heart" combining respiration and contraction compensation in clinical myocardial perfusion SPECT imaging. J Nucl Cardiol 2009;16:396-404.

5. Livieratos L, Rajappan K, Stegger L, Schafers K, Bailey DL, Camici PG. Respiratory gating of cardiac PET data in list-mode acquisition. Eur J Nucl Med Mol Imag 2006;33:584-8.

6. Martinez-Moller A, Zikic D, Botnar RM, Bundschuh RA, Howe W, Ziegler SI, et al. Dual cardiac-respiratory gated PET: Implementation and results from a feasibility study. Eur J Nucl Med Mol Imag 2007;34:1447-54.

7. Pitman AG, Kalff V, Van Every B, Risa B, Barnden LR, Kelly MJ. Contributions of subdiaphragmatic activity, attenuation, and diaphragmatic motion to inferior wall artifact in attenuation-corrected
Tc-99m myocardial perfusion SPECT. J Nucl Cardiol 2005;12: 401-9.

8. Herzog BA, Buechel RR, Katz R, Brueckner M, Husmann L, Burger IA, et al. Nuclear myocardial perfusion imaging with a cadmium-zinc-telluride detector technique: Optimized protocol for scan time reduction. J Nucl Med 2010;51:46-51.

9. Buechel RR, Herzog BA, Husmann L, Burger IA, Pazhenkottil AP, Treyer V, et al. Ultrafast nuclear myocardial perfusion imaging on a new gamma camera with semiconductor detector technique: First clinical validation. Eur J Nucl Med Mol Imag 2010;37:773-8.

10. Ruskin J, Bache RJ, Rembert JC, Greenfield JC Jr. Pressure-flow studies in man: Effect of respiration on left ventricular stroke volume. Circulation 1973;48:79-85.

11. Brenner JI, Waugh RA. Effect of phasic respiration on left ventricular dimension and performance in a normal population. An echocardiographic study. Circulation 1978;57:122-7.

12. Summer WR, Permutt S, Sagawa K, Shoukas AA, BrombergerBarnea B. Effects of spontaneous respiration on canine left ventricular function. Circ Res 1979;45:719-28.

13. Olsen CO, Tyson GS, Maier GW, Davis JW, Rankin JS. Diminished stroke volume during inspiration: Areverse thoracic pump. Circulation 1985;72:668-79.

14. Kim BH, Ishida Y, Tsuneoka Y, Matsubara N, Hiraoka T, Takeda $\mathrm{H}$, et al. Effects of spontaneous respiration on right and left ventricular function: Evaluation by respiratory and ECG gated radionuclide ventriculography. J Nucl Med 1987;28:173-7.

15. Wandt B, Bojo L, Wranne B. Long and short axis dimensions of the left ventricle change in opposite ways during respiration. Acta Physiol Scand 1998;162:9-12.

16. Caiani EG, Turiel M, Muzzupappa S, Porta A, Baselli G, Pagani $\mathrm{M}$, et al. Evaluation of respiratory influences on left ventricular function parameters extracted from echocardiographic acoustic quantification. Physiol Meas 2000;21:175-86.

17. Chen J, Garcia EV, Folks RD, Cooke CD, Faber TL, Tauxe EL, et al. Onset of left ventricular mechanical contraction as determined by phase analysis of ECG-gated myocardial perfusion SPECT imaging: Development of a diagnostic tool for assessment of cardiac mechanical dyssynchrony. J Nucl Cardiol 2005;12: 687-95.

18. Henneman MM, Chen J, Ypenburg C, Dibbets P, Bleeker GB, Boersma E, et al. Phase analysis of gated myocardial perfusion single-photon emission computed tomography compared with tissue Doppler imaging for the assessment of left ventricular dyssynchrony. J Am Coll Cardiol 2007;49:1708-14.

19. Bitarafan-Rajabi A, Rajabi H, Rastgou F, Sharafi AA. Effect of respiratory motion on quantitative myocardial gated SPECT: A simulation study. Ann Nucl Med 2009;23:587-93.

20. Hesse B, Lindhardt TB, Acampa W, Anagnostopoulos C, Ballinger J, Bax JJ, et al. EANM/ESC guidelines for radionuclide imaging of cardiac function. Eur J Nucl Med Mol Imag 2008; 35:851-85.

21. Hesse B, Tagil K, Cuocolo A, Anagnostopoulos C, Bardies M, Bax $\mathrm{J}$, et al. EANM/ESC procedural guidelines for myocardial perfusion imaging in nuclear cardiology. Eur J Nucl Med Mol Imag 2005;32:855-97.

22. Pfisterer ME, Battler A, Zaret BL. Range of normal values for left and right ventricular ejection fraction at rest and during exercise assessed by radionuclide angiocardiography. Eur Heart J 1985;6: 647-55. 